\title{
SÍNDROME DE BURNOUT EN AUXILIARES DE ENFERMERÍA
}

(Burnout syndrome in auxiliary nursing)

Álvaro Luis Fajardo Zapata, M.Sc'

afajardo@areandina.edu.co

Fecha de recepción: 18 noviembre de 2014. Fecha de aceptación: 16 marzo de 2015.

Introducción: El síndrome de Burnout es un complejo de signos y síntomas que se expresan en razón a la exposición a agentes que generan estrés a la que está sometido el trabajador. Objetivo: determinar si un grupo de auxiliares de enfermería que cursan estudios superiores presentan el síndrome de Burnout. Materiales y métodos: estudio exploratorio descriptivo transversal; se aplicó el cuestionario Maslach Burnout Questionnaire (MBI); se realizó un muestreo no probabilístico de tipo intencional; participaron ciento treinta y cuatro auxiliares de enfermería. Resultados: el agotamiento emocional, la despersonalización y la mínima realización personal como indicadores de riesgo de estrés mostraron niveles muy bajos. Quienes presentaron el mayor riesgo de estrés evaluados en los tres ítems fueron las auxiliares mayores de treinta y cinco años. Conclusiones: las auxiliares de enfermería que cursan estudios superiores en enfermería no presentaron indicadores que pudieran hacer pensar que en el momento en que se realizó el estudio estuvieran presentando niveles altos de estrés.

Palabras clave: Despersonalización, Estrés laboral, Auxiliar de enfermería, Riesgo laboral, Burnout profesional, Agotamiento profesional.

Abstract

Introduction: The Burnout syndrome is a complex myriad of signs and symptoms that occurs due to the worker's exposure to stress causing agents. Objective: To determine if a group of auxiliary nurses who are coursing Higher Education Studies present Burnout syndrome. Materials and methods: Descriptive, exploratory and cross-sectional study. The Maslach Burnout questionnaire (MBI) was applied. An intentional non-probability sampling with I34 nursing auxiliaries was carried out. Results: The emotional exhaustion, the depersonalization and the personal fulfillment as indicators of stress risk showed very low levels. Participants with ages above 35 presented the highest risk of stress when the three items were evaluated. Conclusions: Nursing auxiliaries coursing higher education studies in this area, did not present any indicator that leads to think they were presenting high levels of stress in the moment this research was taking place.

Keywords: Depersonalization, Burnout professional, Nursing auxiliary, Labor risk.

' Médico cirujano, ingeniero Industrial, magíster en Toxicología, especialista en Salud Ocupacional. Candidato a Magíster en Salud Pública y Desarrollo social. Profesor asociado del Departamento de Ingeniería y Ciencias Básicas de la Fundación Universitaria del Área Andina, Bogotá, Colombia. 


\section{INTRODUCCIÓN}

Las altas demandas laborales para lograr mayores niveles de productividad y eficiencia por parte de los trabajadores siempre han sido uno de los cuestionamientos que se ha hecho a las empresas. Estas exigencias, de acuerdo con los empleados, no han estado siempre acompañadas ni de los recursos adecuados ni de unas condiciones dignas para desarrollar las labores encomendadas. Las condiciones laborales no adecuadas son una amenaza para el trabajador, ya que pueden generar en él alteraciones orgánicas o psicológicas que afectan su rendimiento $y$, por lo tanto, el de las organizaciones donde este desarrolla sus actividades. Entre las enfermedades asociadas al estrés, generado por las actividades laborales, es posible distinguir un síndrome con características particulares llamado Burnout o 'síndrome de quemarse por el trabajo' (Ponce, Bulnes et al., 2005 y Marrau, 2004).

El síndrome de Burnout es un complejo de signos y síntomas que se expresan en razón a la exposición a agentes que generan estrés a la que está sometido el trabajador y que se expresa clínicamente como un desgaste emocional que afecta la salud y el entorno de quien lo padece. Las investigaciones realizadas en este campo señalan que son los profesionales asistenciales quienes están más expuestos a padecer el síndrome, ya que tienen contacto directo e interactúan con las enfermeras, los médicos, los policías, los psicólogos y los docentes, entre otros (Rubio, 2003).

Maslach propugna que el Burnout se configura como un cuadro tridimensional en el que están inmersos el agotamiento emocional (la sensación de mucho esfuerzo físico y la disminución emocional), la despersonalización (desarrollo de conductas negativas y de insensibilidad) y la mínima realización personal (evaluación propia muy negativa del trabajo realizado y un trato insolente e hipócrita con las personas). De acuerdo con estos autores, las personas jóvenes son más propensas a padecerlo; el género no se ha establecido como un predictor, encontrándose resultados dispares; el estado civil es un factor importante, ya que las personas solteras (especialmente los hombres) lo padecen más que las que viven en pareja; los rasgos de personalidad como baja autoestima y la actitud frente al trabajo pueden contribuir de manera importante. Por último, se presenta más Burnout en las personas con mayor nivel educativo, posiblemente debido a que ocupan puestos con mayor responsabilidad en los que se manejan mayores niveles de estrés, o en los que se tienen mayores expectativas sobre el trabajo, ya que lo que resulta más estresante es que dichas expectativas no se cumplan (Párraga, 2005; Anadón, 2005; Maslach y Léiter, 200I; Maslach, 2003, y Serrano y Moya, 2006).

El Burnout tiene consecuencias importantes tanto para el individuo como para la organización y puede ser altamente costoso. Para alguien que lo padece, lo que antes era importante $y$ significativo, se vuelve desagradable, insatisfactorio y carente de significado. Por otra parte, cuando las personas expresan estas emociones negativas se deterioran las relaciones sociales en el trabajo; las conversaciones se cargan de hostilidad e irritación, por lo que pueden reducirse solamente a temas operativos; también, el trabajo en equipo se desintegra, ya que la hostilidad lleva a que los miembros se distancien y se tienda a evitar en especial a aquellas personas que lo sufren (Maslach y Leiter, 200I; Gil, 2002; lacovides, Fountoulakis et al. 2003, y Manassero, Vásquez et al. 2003).

El síndrome de Burnout afecta la vida de las personas a distintos niveles: somático, psicológico, y/o comportamental. Los episodios y las experiencias de estrés pueden llegar a producir consecuencias perjudiciales para la salud, aunque todavía hay pocos estudios que respalden esta asociación (Pickering, 200I; Steptoe, 200I; Grossi, Perski et al., 2003; De Vente, Olff et al., 2003; Melamed, Ugarten et al., 1999; Roberts, Wessely et al., 2004; Moch, Panz et al., 2003; Bargellini, Barbieri et al., 2000, y Lerman, Melamed et al., 1999). 
Los trabajadores de la salud constituyen uno de los colectivos profesionales que experimentan mayores niveles de estrés laboral y sus consecuencias negativas. Según la Agencia Europea para la Salud, un $28 \%$ de los trabajadores de la sanidad padecen estrés laboral (Arias, Barboza et al., 2007; Chacón y Grau, 1997; Albaladejo, Villanueva y Ortega, 2004, y Cogollo, Batista et al., 20I0).

Actualmente existen múltiples investigaciones que abordan la presencia del estrés laboral crónico o Burnout en profesionales de enfermería; todas ellas concluyen que esto es el resultado de un cúmulo de factores estresantes asociados al desempeño de la profesión y que sobrepasan los recursos de afrontamiento personal (Arias, Barboza et al., 2007; Melita, Cruz y Merino, 2008; Pereda, Márquez et al., 2009, y Bernaldo y Labrador, 2008).

Esta investigación pretende realizar un acercamiento a la realidad del desempeño laboral del personal de enfermería, conocer sus niveles de desgaste psíquico en el trabajo e identificar las principales dificultades que encuentran para el desarrollo satisfactorio de sus funciones. Conocer si existe el síndrome del "quemado" por estrés laboral asistencial en las auxiliares de enfermería puede permitir formular estrategias académicas de intervención en estos grupos de futuros profesionales del área de la salud.

\section{Materiales y MÉTOdos}

Este estudio exploratorio descriptivo de corte transversal consistió en identificar, mediante la aplicación del cuestionario Maslach Burnout Questionnaire (MBI), el cansancio emocional $(\mathrm{AE})$, la despersonalización (DP) y la realización personal (RP). Las variables sociodemográficas investigadas fueron: (a) edad, (b) estado civil, (c) cantidad de hijos, (d) antigüedad en la profesión y (e) turno de trabajo. Como universo se tomó la totalidad de las auxiliares de enfermería que estuvieran cursando estudios superiores en Enfermería en una institución universitaria (594 estudiantes).
No se realizó ninguna intervención durante el desarrollo del estudio. El tamaño de la muestra fue de 134 encuestas, realizadas mediante muestreo no probabilístico de tipo intencional. Se tomó como error el $7 \%$, y una prevalencia estimada del $27 \%$. Se excluyeron del estudio los hombres, tanto como quienes tuvieran menos de dos años de estar laborando como auxiliares de enfermería; también se excluyeron las encuestas que no estuvieran completamente diligenciadas. El manejo de la información fue anónimo y confidencial.

Los datos producto de la investigación fueron ingresados en bases de datos específicas. Estas se elaboraron en Excel y los análisis se realizaron en Epiinfo 3.3.2.

\section{Resultados}

El $58 \%$ de las mujeres encuestadas tenían edades comprendidas entre 25 y 34 años; el 26 $\%$, entre 20 y 24 años y solamente el $16 \%$ más de 35 años. El $56 \%$ de ellas eran solteras; el $21 \%$, separadas; el $15 \%$, vivían en unión libre y el $8 \%$ eran casadas. En cuanto al número de hijos, el $39 \%$ de ellas tenían un hijo, el $36 \%$, no tenía hijos; el $21 \%$, dos hijos; el $3 \%$, tres y el I \%, cuatro.

El $30 \%$ de las auxiliares de enfermería del presente estudio tenía una experiencia laboral de 2 a 4 años; el $29 \%$, de 5 a 7 años; el $26 \%$, de 8 a 10 años y el $15 \%$, más de 10 años. El 51 $\%$ trabajaba en el turno del día, mientras que el 49 \% lo hacía en el turno de la noche.

Para el ítem de agotamiento emocional como indicador de estrés, el estudio mostró que el 46 $\%$ de las auxiliares de enfermería presentaba un riesgo bajo, el $33 \%$, un riesgo medio y el 21 $\%$, riesgo elevado. Para el ítem de despersonalización, el 33 \% presentó un riesgo bajo; el 37 $\%$ un riesgo medio y el $30 \%$ riesgo elevado. Para el ítem de realización mínima personal, el $67 \%$ presentó un riesgo bajo; el $19 \%$ un riesgo medio y el $14 \%$ riesgo elevado. 
Analizado por grupo etario, el estudio mostró que el agotamiento emocional como indicador de riesgo de estrés fue bajo (46,5\%); el grupo que tuvo el menor riesgo fue el de 25 a 29 años $(29,5 \%)$ y el que tuvo el mayor riesgo fue el de más de 35 años (15,5\%).

El factor de despersonalización como indicador de riesgo de estrés fue medio ( $37,2 \%)$; el grupo que tuvo el menor riesgo fue el de 25 a 29 años $(29,5 \%)$, y el que tuvo el mayor riesgo fue el de más de 35 años (I5,5\%). Asimismo, el ítem de realización mínima personal como indicador de riesgo de estrés fue bajo (66,7\%); el grupo que presentaba el menor riesgo fue el de 25 a 29 años (29,5\%) y el que tuvo el mayor riesgo fue el de más de 35 años (I5,5\%). No se encontró asociación entre el grupo etario, el agotamiento emocional, la despersonalización y la realización personal.

El análisis de la experiencia laboral, en tanto riesgo para el agotamiento emocional, como indicador de estrés, fue bajo (46,5\%); el grupo que tuvo el menor riesgo fue el de 2 a 4 años $(30,2 \%)$ y el que tuvo el mayor riesgo fue el de más de 35 años (I5,5\%). En cuanto al factor despersonalización, como indicador de estrés, fue medio (37,2 \%); el grupo que tuvo el menor riesgo fue el de 2 a 4 años (30,2 \%) y el de ma- yor riesgo fue el de más de 35 años (15,5\%). Asimismo, la experiencia laboral, en tanto riesgo para la realización personal, como indicador de estrés, fue bajo (66,7 \%); el grupo que tuvo el menor riesgo fue el de 2 a 4 años (30,2\%) y el mayor riesgo lo presentaron las mujeres de más de 35 años (15,5\%). No se encontró asociación entre la experiencia laboral, el agotamiento emocional, la despersonalización y la realización personal.

El análisis del estado civil, en tanto riesgo para el agotamiento emocional, como indicador de estrés, fue bajo (46,5 \%); fueron las mujeres solteras las que tuvieron el menor riesgo (47,3 \%) y las que tuvieron el mayor riesgo fueron las mujeres separadas (13,2\%). La despersonalización, en tanto indicador de estrés, fue medio (37,2 \%); las mujeres solteras tuvieron el menor riesgo $(47,3 \%)$ y las que tuvieron el mayor riesgo fueron las mujeres separadas. $\mathrm{La}$ realización personal, como indicador de estrés, fue bajo (66,7 \%); fueron las solteras las que tuvieron el menor riesgo $(47,3 \%)$ y el mayor riesgo fue para las mujeres separadas (13,2 $\%)$. No se encontró asociación entre el estado civil, el agotamiento emocional, y la despersonalización. Estos resultados se resumen en la Tabla I, que se presenta a continuación.

\begin{tabular}{|l|l|l|l|}
\hline FACTOR DE RIESGO & \multicolumn{1}{|c|}{$\begin{array}{c}\text { AGOTAMIENTO } \\
\text { EMOCIONAL }\end{array}$} & \multicolumn{1}{|c|}{ DESPERSONALIZACIÓN } & \multicolumn{1}{|c|}{$\begin{array}{c}\text { REALIZACIÓN MíNIMA } \\
\text { PERSONAL }\end{array}$} \\
\hline EDAD & Riesgo bajo & Riesgo medio & Riesgo bajo \\
\hline EXPERIENCIA LABORAL & Riesgo bajo & Riesgo medio & Riesgo bajo \\
\hline ESTADO CIVIL & Riesgo bajo & Riesgo medio & Riesgo bajo \\
\hline
\end{tabular}

Tabla I. Análisis de hallazgos por factor de riesgo (Fuente: Autor, 2014). 
Analizados cada uno de los indicadores para evaluar el estrés en las auxiliares de enfermería, el presente trabajo de investigación encontró que el indicador para agotamiento emocional presentó un $21 \%$ de riesgo elevado, un $30 \%$ de riesgo elevado para la despersonalización y un $14 \%$ de riesgo elevado para el indicador de realización personal.

En cuanto al grupo etario, el estudio mostró que el agotamiento emocional, la despersonalización y la realización personal, como indicadores de riesgo de estrés, se acentuaron más en el grupo de las mujeres mayores de 35 años. No se encontró asociación entre el grupo etario y el agotamiento emocional, la despersonalización y la realización personal.

La experiencia laboral, en tanto riesgo para el agotamiento emocional, la despersonalización y la realización mínima personal, como indicadores de estrés, también se presentaron de manera acentuada en el grupo de mujeres mayores de 35 años de edad, sin que se encontrara asociación en ninguno de los tres indicadores y la experiencia laboral.

El análisis del estado civil, en tanto riesgo para el agotamiento emocional, la despersonalización y la realización mínima personal como indicadores de estrés, encontró que son las mujeres separadas las que tienen el mayor riesgo de padecerlo, sin que se hallara asociación entre estos tres indicadores y el estado civil.

\section{DisCUSIÓN}

De acuerdo con los hallazgos de este estudio, y tomando en consideración los criterios de la escala de Burnout para el análisis de riesgo para estrés, las auxiliares de enfermería, que cursan estudios superiores en enfermería, no presentaron indicadores que pudieran hacer pensar que en el momento en que se realizó la investigación estuvieran presentando niveles altos de estrés; sin embargo, a pesar de que los resultados mostraron bajos niveles de estresores, evaluadas estas mismas personas en otro tiempo, estos indicadores puedan mostrar resultados completamente diferentes.

Es importante anotar que quienes presentaron el mayor riesgo de estrés, evaluados los tres ítems: agotamiento emocional, despersonalización y realización personal, fueron las auxiliares de enfermería mayores de 35 años, contrario a lo encontrado en los estudios de Maslach, Leitter y Serrano, quienes hallaron que las personas jóvenes son más propensas a padecer este síndrome. Los resultados del presente estudio coinciden con los encontrados por Francisco Labrador, quien afirma haber descubierto un incremento en la aparición del síndrome de Burnout relacionado con la mayor edad y la antigüedad en el puesto de trabajo de la enfermera (Maslach, 200I; Serrano y Moya, 2006, y Bernaldo y Labrador, 2008).

El nivel educativo y el género como factores comparativos no fueron considerados como variables en el presente estudio, ya que todos los participantes fueron mujeres, técnicas en enfermería. Es posible que si se realiza un trabajo posterior en el que participen tanto auxiliares de enfermería, como enfermeras jefes y hombres, se puedan encontrar diferencias.

Con relación al horario laboral, este estudio no encontró ninguna diferencia entre el turno diurno y el nocturno que pudiera hacer pensar que esta variable afecte de manera importante a este grupo de trabajadoras de la salud, aunque se afirma que la disponibilidad horaria puede modificar los ritmos circadianos, alterar el sueño y tener repercusiones sobre la vida familiar $y$ social (Chacón y Grau, 1997).

Conflicto de intereses: Ninguno \& 
Referencias bibliográficas

Albaladejo, R., Villanueva, R. y Ortega, P. (2004). Síndrome de Burnout en el personal de enfermería de un hospital de Madrid. Revista Española de Salud Pública, 78(4).

Anadón, O. (2005). La Formación en estrés para la prevención del síndrome de «Burnout» en el Currículo de Formación Inicial de los maestros. Revista Interuniversitaria de Formación del Profesorado, I9(I), 197-220.

Arias, F., Barboza, N., Blanco, J., Fajardo, E., Rivera, R., Rodríguez, E., Ugalde, S. y Lépiz, R. (2007). Síndrome del desgaste profesional o de Burnout en el personal de enfermería. Revista Enfermería Actual en Costa Rica (en línea). Recuperado en marzo de 2013, de: http://www. revistas.ucr.ac.cr/index.php/enfermeria/article/view/3600/3508

Bargellini, A., Barbieri, A., Rovesti, S., Vivoli, R., Roncaglia, R. y Borella, P. (2000). Relation between immune variables and burnout in a sample of physicians. Journal of Occupational and Environmental Medicine, 57, 453-457.

Bernaldo, M. y Labrador, F. (2008). Relaciones entre estrés laboral, Burnout y síntomas psicopatológicos en los servicios de urgencia extrahospitalaria del Área 9 de Madrid. Anuario de Psicología Clínica y de la Salud, 4, 53-6I.

Chacón, M. y Grau, J. (1997). Burnout en enfermeros que brindan atención a pacientes oncológicos. Revista Cubana de Oncología, I3(2), II8-I 25.

Cogollo, Z., Batista, E., Cantillo, Ch., Jaramillo, A., Rodelo, D. y Meriño, G. (2010). Desgaste profesional y factores asociados en personal de enfermería de servicios de urgencias de Cartagena, Colombia. Aquichan, I0(I), 43-5I.

De Vente, W., OIff, M., Van Amsterdam, J., Kamphuis, J. y Emmelkamp, P. (2003). Physiological differences between Burnout patients and healthy controls: blood pressure, heart rate, and cortisol responses. Occupational \& Environmental Medicine, 60(I), 54-6I.

Gil, P. (2002). Síndrome de quemarse por el trabajo: aproximaciones teóricas, explicación y recomendaciones para su intervención (documento elaborado Para la OMS). Revista electrónica de Psicología Científica (en línea). Recuperado en marzo de 2013, de: http://www. psicologiacientifica.com/?s=s\%C3\%ADndrome\&submit=Buscar

Grossi, G., Perski, A., Evergard, B., Blomkvist, V. y Orth-Gomér, K. (2003). Physiological correlates of Burnout among women. Journal of Psychosomatic Medicine, 55, 306-316.

lacovides, A., Fountoulakis, K., Krapinis, S. y Kaprinis, G. (2003). The relationship between job stress, Burnout and clinical depression. Journal of Affective Disorders, 75, 209-22I.

Leiter, M. y Maslach, C. (200I). Burnout and Quality in a Sped-Up World. The Journal for Quality and Participation, 24(2), 48-5 I.

Lerman, Y., Melamed, S., Shragin, Y., Kushnir, T., Rotgoltz, Y., Shirom, A. y Aronson, M. (1999). Association between Burnout at work and leukocyte adhesiveness/aggregation. Psychosomatic Medicine, 6I, 828-833.

Manassero, M., Vázquez, A., Ferrer, V., Fornés, J. y Fernández, M. (2003). Estrés y Burnout en la enseñanza. Recuperado en abril de 2013, de http://www.redalyc.org/pdf/23I3/231317039006.pdf

Marrau, C. (2004). El síndrome de Burnout y sus posibles consecuencias en el trabajador docente. Fundamentos de humanidades, 2(I0), 53-68.

Maslach, C., Leiter, P. y Schaufeli, W. (200I). Job Burnout. Annual Review of Psychology, 52, 397- 422.

Maslach, C. (2003). Job Burnout: New Directions in Research and Intervention. American Psychological Society, I2(5), I89-192.

Melamed, S., Ugarten, U., Shirom, A., Kahana, L., Lerman, Y. y Froom, P. (1999). Chronic Burnout, somatic arousal and elevated cortisol levels. Journal of Psychosomatic Research, 6, 591-598.

Melita, A., Cruz, M. y Merino, J. (2008). Burnout en profesionales de enfermería que trabajan en centros asistenciales de la Octava Región de Chile. Ciencia y Enfermería, 24(2),75-85.

Moch, S., Panz, V., Joffe, B., Havlik, I. y Moch, J. (2003). Longitudinal changes in pituitary-adrenal hormones in South African women with Burnout. Endocrine, 2I(3), 267-272.

Párraga, J. (2005). Eficacia del programa I.R.I.S. para reducir el síndrome Burnout y mejorar las disfunciones emocionales en profesionales sanitarios (Tesis doctoral). Universidad de Extremadura, Madrid. Recuperada de: http://dialnet.unirioja.es/servlet/tesis?codigo $=595$

Pereda, L., Márquez, F., Hoyos, M. y Yánez, M. (2009). Síndrome de Burnout en médicos y personal paramédico. Salud Mental, 32(5), 399-404.

Pickering, T. (200I). Job stress, control, and chronic disease: moving to the next level of evidence. Psychosomatic Medicine, 63, 734-736. 
Ponce, C., Bulnes, M., Aliaga, J., Atalaya, M. y Huertas, R. (2005). El síndrome del "quemado" por estrés laboral asistencial en grupos de docentes universitarios. Revista IIPSI, de la Facultad de Psicología UNMSM, 8(2), 87-I I 2.

Riveri, R., Sánchez, A., Castellanos, M., Jay, B. y Ricardo, C. (2008). Síndrome de Burnout en el personal de enfermería del Servicio de Terapia Polivalente Hospital Saturnino Lora. Revista Cubana de Enfermería, 24(2).

Roberts, A., Wessely, S., Chalder, T., Papadopoulos, A. y Cleare, A. (2004). Salivary cortisol response to awakening in chronic fatigue syndrome. British Journal of Psychiatry, 184, I36-I4I.

Rubio, J. (2003). Fuentes de estrés, Síndrome de Burnout y actitudes disfuncionales en orientadores de Instituto de Enseñanza Secundaria (Tesis doctoral). Universidad de Extremadura, Badajoz, España. Recuperada de:

file://C:/Users/usuario I/Downloads/Dialnet-FuentesDeEstresSindromeDeBurnoutYActitudesDisfunci-I494.pdf

Serrano, R. y Moya, M. A. (2006). Adaptación psicobiológica al estrés social en una muestra de profesores: cambios hormonales, cardiovasculares y psicológicos (Tesis doctoral). Universidad de Valencia, España. Recuperada en:

http://www.tdx.cat/bitstream/handle/ I0803/I0 I89/serrano.pdf;jsessionid=77| 3FB6 I4AFI3DD74A3D9A328 I 9CC75B.tdx2?sequece = I

Steptoe, A. (200I). Job control, perceptions of control, and cardiovascular activity: an analysis of ambulatory measures collected over the working day. Journal of Psychosomatic Research, 50, 57-63. 